\title{
Guidelines for the Functional Analysis of Engineered and Mutant Enzymes
}

\author{
Dale E. Edmondson and Giovanni Gadda
}

\section{1 Introduction}

The basic goal of protein engineering is the creation of altered forms of a known enzyme catalyst that exhibits one or more of the following properties:

- An increased catalytic function relative to the parent enzyme.

- An altered substrate specificity or stereospecificity, such that the engineered protein is capable of catalyzing the conversion of substrates differing from the specific substrate of its parent form.

- An increased stability to the environment that is required for it to catalyze the specific function required.

An analysis of all of these desired properties of the engineered protein requires detailed studies of its catalytic properties, which involve the delineation of the steady-state kinetic behavior of the mutant protein. This chapter deals with topics ranging from the acquisition of data, the analysis of the kinetic data acquired, and the interpretations and conclusions that may be inferred from those data. This chapter is not meant to be a reiteration of chapters and textbooks on enzyme kinetic approaches that are either classics in the field or have been recently published. Rather, it is meant for readers who have attended a basic biochemistry course in which enzyme kinetics is only cursorily presented. Hopefully, this chapter will fill a gap between an introductory level and the more rigorous treatments of the subject which are written for readers well versed in the field. The aim here is to describe approaches that the authors have used over their careers in order to provide a readable and useful 'road map' for those colleagues and students who have either not been exposed to this area of enzymology, are just entering the field, or have not had time to 'wade through' those texts that have been published in the area of enzyme kinetics [1, 2]. This chapter is organized as follows: a review of steady-state kinetic equations, consideration of assay procedures, interpretation of basic kinetic parameters, the effect of $\mathrm{pH}$ on enzyme 
kinetic parameters, and an introduction to enzymes catalyzing reactions that require two substrates.

\section{2}

\section{Steady-State Kinetics}

In order to appreciate the rest of the topics covered in this chapter, a brief review of the steady-state kinetic approach and the parameters of enzyme-catalyzed reactions is in order. The basic equation describing enzyme catalysis is the Michaelis-Menten equation:

$$
\frac{v_{o}}{[E]}=\frac{k_{\mathrm{cat}}[S]}{K_{m}+[S]}
$$

where $k_{\text {cat }}$ (units of time $e^{-1}$ ) is the enzyme turnover number, which is defined as the maximum number of substrate molecules converted to products per active site per unit of time (with units of a first-order rate constant). $K_{\mathrm{m}}$ (units of concentration) is defined as the concentration of substrate where half the maximal activity $\left(k_{\text {cat }}\right)$ is observed. The term $v_{0}$ defines the initial, zero-order rate of the reaction (with units of concentration of product-time ${ }^{-1}$ ), the value of which depends hyperbolically on the substrate concentration.

The requirements for the valid application of the Michaelis-Menten equation are the following:

1. [S] must be significantly larger than [E] (ideally at least 100-fold).

2. The steady-state assumption holds (where the concentration of ES is constant over the course of the assay) and the rate of product formation is linear with time.

3. The initial rate of the reaction is such where the concentration of substrate does not materially change over the course of the assay and the concentration of product is small enough, so that no enzyme inhibition or reverse reaction occurs.

These requirements are readily fulfilled when enzymes catalyzing the transformations of small molecules are studied. Problems arise, however, when Michaelis-Menten kinetic equations are applied to enzymes catalyzing the transformations of large macromolecules, such as other proteins or polynucleotides. The lack of fulfillment of any of the three requirements summarized above prevents the valid application of the Michaelis-Menten equation, and requires the application of other, specialized kinetic approaches that are dependent on the nature of the system examined.

Under conditions in which the concentration of substrate is significantly smaller than the $K_{\mathrm{m}}$ value (normally at least 10 -fold smaller), the Michaelis-Menten equation simplifies to that designated in Equation 1.2. 


$$
\frac{v_{0}}{[E]}=\frac{k_{\mathrm{cat}}}{K_{m}}[S]
$$

In this form the novel kinetic parameter is $k_{\text {cat }} / K_{\mathrm{m}}$ [with units of (conc. of substrate $)^{-1}$ time $^{-1}$ ], which is often referred to as a measure of 'catalytic efficiency' of the engineered enzyme under study. In this condition, the initial velocity is linearly dependent on the substrate concentration and the slope of this linear relation is $k_{\text {cat }} / K_{\mathrm{m}}$, which has the units of a second-order rate constant.

\section{3}

Enzyme Assays and the Acquisition of Initial Velocity Data

\subsection{1}

\section{Biological Sample Appropriate for Assay}

The authors assume that the investigator has gone through the construction and expression of the engineered enzyme. (Other chapters in this series deal with the details of that aspect.) The question being considered here is whether it is sufficient to use crude extracts of the expression system producing the desired engineered enzyme, whole cells containing the enzyme, or should one go through the tedium of purification of the engineered enzyme for acquisition of kinetic data. There are various views on this topic. If the investigator chooses to use whole cells that contain the engineered protein as enzyme source, there are considerable pitfalls. In screening constructs, the assay of whole cells is a requirement in screening for cells containing the desired clones. Once the desired clones have been isolated and detailed properties of the desired mutant protein are measured, then there are considerable dangers in determining the kinetic properties in either whole cells or crude extract systems. The problem of substrate not being able to penetrate the cell wall is the most detrimental aspect of assaying activity in whole-cell assays. In addition, there is no good measure of the level of functional protein expressed, so that determination of relative values of $k_{\text {cat }}$ is problematic, as $k_{\text {cat }}=V_{\max } /[$ enzyme $]$. The immunochemical detection of the expressed protein in cell lysates measures the total amount of expressed protein, but does not provide a quantitative level of functional protein expressed. In addition to these difficulties, there is always the possibility that endogenous enzyme systems in the expression host may react with the substrate of interest (even though the reaction catalyzed may differ), and therefore provide inaccurate velocity data if the assay relies on the loss of substrate as a function of time as a measure of the enzyme activity. With the availability of rapid and easy purification systems for expressed enzymes through the use of polyhistidine tags, or other tags engineered into the protein at either the amino or carboxyl terminus, the use of purified enzymes offers all of the advantages with none of the problems outlined above in the determination of its substrate specificity and kinetic properties. One potential difficulty in the use 
of purified enzymes (especially if they are membrane-bound) is the possibility of their reduced stability on purification, which might necessitate the addition of reagents to stabilize them. In many cases, the addition of glycerol $(5-20 \%, v / v)$ to stabilize a solution of purified enzyme has been a useful approach for the storage and handling of active and stable enzymes.

\section{3 .2}

\section{Enzymatic Assays}

The quantitative determination of enzymatic activity is a diverse topic and boils down to an exercise in analytical chemistry. In general, enzyme assays can be divided into two approaches; a discontinuous assay and a continuous assay. The difference is that the former approach is more tedious while the latter is more convenient, allowing the rapid acquisition of kinetic data, and is applicable for the use of high-throughput assay techniques. In both approaches, every effort should be made to develop methodology for monitoring the formation of product as a function of time rather than the decrease in the concentration of substrate concentrations. Basic to this question is one of accuracy. In the case of product formation, one begins with a 'zero' concentration and ends up with a finite amount of product formed. Therefore, one is looking at a difference (with respect to time) of a finite numerical value versus a zero value. In the case where one observes substrate loss as a function of time, the difference in determinations of substrate concentrations then involves differences in two rather large numbers, with the result being a difference in concentration of substrate that incorporates the errors involved in the analytical determinations of the amount of substrate present at various time periods in the assay. The conclusion from this discussion is that, whenever possible, it is preferable to assay the activity of enzymes by product analysis rather than by substrate analysis.

In the discontinuous enzyme assay, aliquots are removed from the incubation mixture of enzyme plus substrate at various times and product formation is determined by separation techniques that range from solvent extraction to chromatographic separations such as high-performance liquid chromatography (HPLC). The advantage of radiochemical tagging of substrate so that product concentrations are readily determined (e.g. ${ }^{14} \mathrm{C}$ labeling at a nonlabile position) is readily apparent, although the execution of this approach requires a source of radiolabeled material of a known specific activity, which may not always be possible. Alternatives to radiolabeling procedures include mass spectrometry (MS) or gas chromatography (GC) if the products of the reaction can be made volatile, either inherently or by suitable derivatization. Most often there exist specific absorption spectral or optical rotation properties of the product that allow its quantitative determination on separation from substrate.

A fundamental issue that requires care when using a discontinuous assay (also referred to as an 'end-point' assay) for the determination of enzymatic activity is that of the linearity of product formation (or substrate depletion) with time at the initial stages of the reaction. This is an essential methodological aspect that is 
required when steady-state kinetic approaches are applied, as the observed rate of the enzymatic reaction under study is given by the slope of the tangent line to the progress curve of the reaction in a plot of (product) versus time-that is, a line that in a discontinuous assay is given by only two points. To ensure that product formation is linear over time, one must therefore repeat the assay under the same experimental conditions and determine the concentration of product at several time points during the course of the reaction-a sometimes tedious, but absolutely required, procedure. Failure to do so can invariably result in an underestimation of the enzymatic activity due to the time point selected for the quantification of the concentration of product not being in the linear portion of the progress curve of the enzymatic assay, as illustrated in the example of Figure 1.1.

The continuous enzyme assay is usually performed by monitoring the changes in absorption or fluorescent spectral properties associated with product formation in the catalytic reaction. The initial rates are determined continuously and the increase in product concentration quantified as a function of time, using the abovedescribed spectral approaches.

In the event that the product does not exhibit a measurable spectral property, a valuable alternative approach is to 'couple' the assay so that formation of product is coupled to a second enzyme system which does provide a signature spectral property that can be measured either by absorption or fluorescence. The investigator should design the coupled assay such that the limiting velocity is due to the enzyme-catalyzed reaction under study and not limited by the coupling enzymes used. A valuable discussion of techniques used in coupled enzyme assays has been published [3]. One example is the formation of $\mathrm{H}_{2} \mathrm{O}_{2}$ from the reduction of $\mathrm{O}_{2}$ in a number of oxidase enzyme systems. The molar absorption of $\mathrm{H}_{2} \mathrm{O}_{2}$ is reasonably weak $\left(\varepsilon_{240 \mathrm{~nm}}=35 \mathrm{M}^{-1} \mathrm{~cm}^{-1}\right)$ and is maximal in the ultraviolet (UV) region, which also contains high, interfering absorption from the enzyme and other substrates

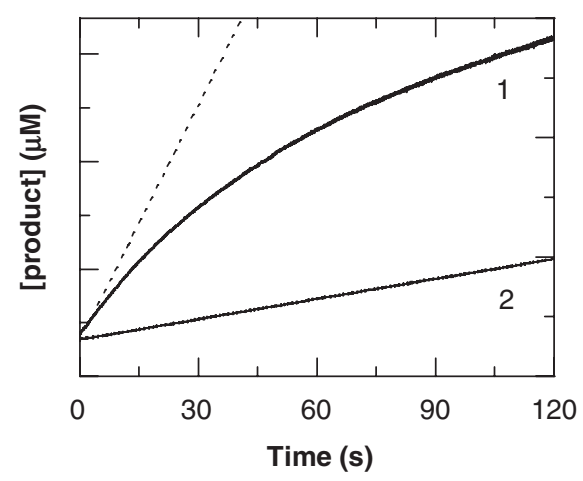

Figure 1.1 Plot of initial rate of product formation with time in an enzyme assay run under zero-order conditions. Line 1 shows the nonlinear appearance of product with time, which is analyzed by the drawing of a tangent (---) to the initial rate of product formation. Line 2 shows a linear rate of product formation with time. 


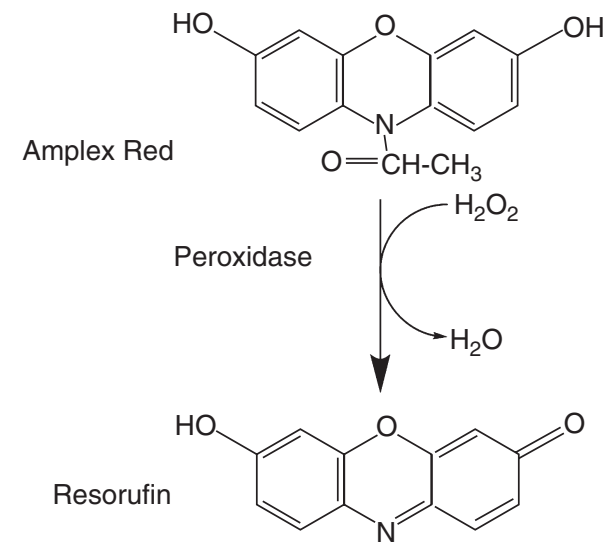

Figure 1.2 Reaction scheme for the coupled enzyme assay for the detection of hydrogen peroxide using Amplex Red/ horseradish peroxidase.

or products. A solution to this problem is to couple the formation of $\mathrm{H}_{2} \mathrm{O}_{2}$ with the enzyme horseradish peroxidase, which uses a chromogenic substrate so that the rate of formation of $\mathrm{H}_{2} \mathrm{O}_{2}$ can be readily monitored. The current peroxidase substrate of choice is Amplex Red, which reacts with $\mathrm{H}_{2} \mathrm{O}_{2}$ in a peroxidasecatalyzed reaction to form resorufin $\left(\varepsilon_{560}=56000 \mathrm{M}^{-1} \mathrm{~cm}^{-1}\right)$, thus giving a highly sensitive method for the detection of $\mathrm{H}_{2} \mathrm{O}_{2}$ formation [4, 5] (Figure 1.2). The assay can be made even more sensitive by the use of fluorescence arising from the resorufin product. A valuable article on the use of horseradish peroxidase-coupled assays and their potential pitfalls has been published by Holt and Palcic [6].

Continuous assays can also be followed by the use of electrochemical detection of substrate and/or products. Common electrodes used include the polarographic Clark electrode for the detection of $\mathrm{O}_{2}$, and glass electrodes for the detection of $\mathrm{H}^{+}$ liberated in hydrolytic reactions. The $\mathrm{O}_{2}$ electrodes suffer from a lack of sensitivity, membrane instability, and functionality over a limited temperature range. In addition, the investigator is dealing with the problem of measuring small differences in $\mathrm{O}_{2}$ concentration by subtraction of two relatively large numerical values, which limits the precision of the measurement. Some of these problems can be overcome by the use of $\mathrm{O}_{2}$ probes which utilize the quenching of a bound fluorophore by paramagnetic ground-state $\mathrm{O}_{2}$ in solution. Care should be exercised to determine that fluorophore fluorescence is not quenched by solution components in addition to dissolved $\mathrm{O}_{2}$.

\subsection{3}

\section{Analysis of Initial Rate Data}

Once protocols are established for the measurement of initial velocity data, the investigator then determines the effect of substrate concentration on the initial rate of the enzymatic reaction. As most enzymes require two substrates, typically 


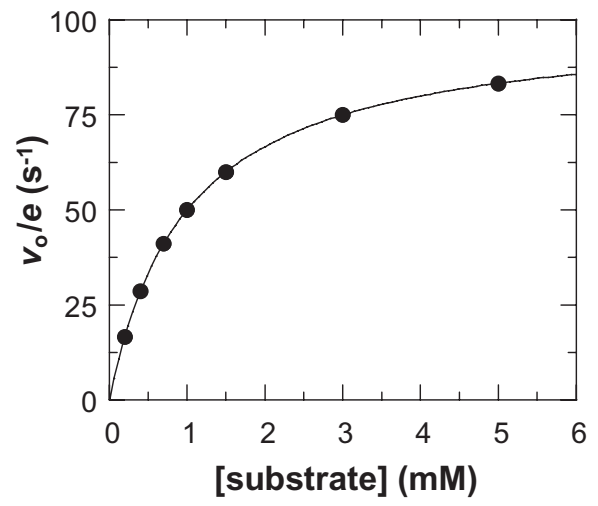

Figure 1.3 Hyperbolic plot of the dependence of the initial rate on the substrate concentration for an enzyme-catalyzed reaction. The data presented are for an enzyme with a $k_{\text {cat }}$ of $100 \mathrm{~s}^{-1}$, a $K_{\mathrm{m}}$ of $1 \mathrm{mM}$, and a range of $[S]=0.2-5 \mathrm{mM}$. The solid curve is a nonlinear fit of the data using Equation 1.1.

one substrate is kept at a fixed concentration while the concentration of the other substrate is varied. The data are plotted using the well known Michaelis-Menten equation (Equation 1.1), which exhibits a hyperbolic dependence of rate $\left(v_{0}\right)$ on [S] (Figure 1.3):

The most accurate method of determining the parameters $k_{\text {cat }}$ and $K_{\mathrm{m}}$ is to fit the hyperbolic relationship by nonlinear fits using commonly available commercial software programs such as Origin, KaleidaGraph or SigmaPlot, all of which are available for use on most personal computers. The data required are initial rates at substrate concentrations ranging from 0.2 - to 5 -fold the $K_{\mathrm{m}}$ value of the enzyme for the substrate, ideally with an equal number of data points below and above the $K_{\mathrm{m}}$ value. Under these conditions, the investigator can determine accurate values for $k_{\text {cat }}, K_{\mathrm{m}}$ and $k_{\text {cat }} / K_{\mathrm{m}}$ for the engineered enzyme of interest. Linearization of the data using the popular Lineweaver-Burk plot $\left(1 / \nu_{\mathrm{o}}\right.$ versus $1 /[\mathrm{S}]$ provides $k_{\text {cat }}, K_{\mathrm{m}}$ and $k_{\text {cat }} / K_{\mathrm{m}}$ values, which are less accurate as the method accentuates uncertainty by giving disproportionate weight to the least accurate data (i.e. low $v_{\mathrm{o}}$ values at low concentrations of substrate). A more accurate linear plot, which provides a more balanced representation of the data, is the Hanes plot ([S]/vo versus $[\mathrm{S}]$ ). For a detailed discussion of the various linear plotting methods, the reader is referred to a very readable treatment by Cornish-Bowden [1]. In the event that the investigator encounters a situation where the solubility of the substrate prevents the use of substrate concentrations required for saturation of the enzyme (i.e. one cannot reach $k_{\mathrm{cat}}$ conditions), both linear and nonlinear fits of the data break down and lead to inaccurate $k_{\text {cat }}$ values (and consequently $K_{\mathrm{m}}$ values). Under these conditions the investigator can only determine $k_{\text {cat }} / K_{\mathrm{m}}$ values for the enzyme by using Equation 1.2, and must restrain from reporting artifactual $k_{\text {cat }}$ and $K_{\mathrm{m}}$ values. In opposite instances where the $K_{\mathrm{m}}$ value is too small to be accurately 
determined, the investigator can only determine the $k_{\text {cat }}$ values, and must refrain from reporting artifactual $k_{\text {cat }} / K_{\mathrm{m}}$ and $K_{\mathrm{m}}$ values.

\section{3 .4}

\section{Determination of Functional Catalytic Site Concentrations}

The definitive determination of $k_{\text {cat }}$ relies on measuring the concentration of functional catalytic sites used in the catalytic assays. This is not a trivial point in the analysis of recombinant engineered enzymes, as purified preparations may contain an unknown fraction of protein molecules that may not be correctly folded or may not contain the native cofactor (if one is required for catalysis). Simply determining the protein concentration and assuming there to be one catalytic site per mole of enzyme is not sufficient for the correct determination of $k_{\text {cat }}$ values. The most direct approach to determine the concentration of functional enzyme is a titration with an irreversible mechanism-based inhibitor that forms a covalent bond to a residue in the active site [7]. This approach is the most practical one for a wide range of enzymes utilizing small-molecule substrates. Assuming that there is no partitioning between turnover and covalent labeling, one simply 'titrates' a solution of recombinant enzyme and determines the active sites concentration from a plot of residual activity versus amount of inhibitor added [8]. In cases where the catalyzed turnover can compete with covalent labeling, one can use a radiolabeled inhibitor and determine covalent incorporation by the amount of radiolabeling on precipitation of the enzyme under denaturing conditions. If no radiolabeled inhibitor is available, and no absorption or fluorescent spectral changes associated with covalent inhibitor incorporation are detectable, the level of covalent inhibitor incorporation can be determined using high-resolution, quantitative electrospray ionization mass spectrometry, where the increase in molecular weight of the enzyme on covalent modification is monitored and the ratio of enzyme inhibitor complex to the amount of unlabeled enzyme can be determined $[9,10]$. However, the latter approach is not easily performed in most laboratories, and requires personnel and equipment that are highly specialized.

If working with enzymes that contain redox-active cofactors, the level of functional enzyme is readily determined by the level of cofactor reduction that occurs rapidly on the anaerobic addition of a reducing substrate. This approach is commonly used with flavoenzymes and metalloenzymes where the metal or flavin coenzyme undergoes a redox change on substrate addition which can be followed by UV-visible absorption spectroscopy in the absence of an oxidizing agent such as $\mathrm{O}_{2}$.

\section{4}

\section{Steady-State Kinetic Parameters and Their Interpretation}

After the efforts involved in expressing and purifying the engineered enzymes and the accurate determination of their steady-state kinetic properties, one is 
left with three numbers: $k_{\text {cat }}, K_{\mathrm{m}}$ and $k_{\text {cat }} / K_{\mathrm{m}}$, as quantitative measures of their functional properties. The value of $k_{\text {cat }}$ is the rate of overall turnover in the reaction catalyzed, and is useful to compare the functional properties of the engineered protein with that of the wild-type enzyme. The catalytic turnover number has the units of $\mathrm{t}^{-1}$, and is usually expressed in $\mathrm{min}^{-1}$ or $\mathrm{s}^{-1}$. The value of $k_{\text {cat }}$ is a composite of all of the kinetic steps involved in catalysis at saturating concentrations of the substrates, and its value is dependent on the value of the reaction step that is the slowest in enzyme turnover and therefore constitutes the rate-limiting step in catalysis. This rate-limiting reaction need not necessarily involve a step involved in the chemical transformation of the substrate to product, but may be given by the rate of product release from the catalytic site. The goal of most enzyme engineering studies is to alter the substrate specificity of the mutated enzyme or to increase the $k_{\text {cat }}$ value. Therefore, the investigator should measure the $k_{\text {cat }}$ values for wild-type and mutant enzymes with the native substrate as well as with the desired specific substrate, with the caution that the rate-limiting step in catalysis observed with the native enzyme may be different for the engineered or mutant form.

The value of $K_{\mathrm{m}}$ is defined as the substrate concentration where $k_{\text {cat }} / 2$ occurs, and is expressed in terms of concentration (usually $\mathrm{m} M$ or $\mu M$ ). Only in special cases is it a measure of substrate binding affinity to the enzyme (i.e. $K_{\mathrm{d}}$ ). In the simplest instance of an irreversible enzyme-catalyzed reaction with a single substrate, the $K_{\mathrm{m}}$ will reflect the enzyme-binding affinity for the substrate only when the rate of the chemical step is significantly slower than both the rates of dissociation of the substrate and the product from the catalytic site. (For an in-depth discussion of this topic, the reader is referred to Ref [3] and [4].) The special case described above would require extensive kinetic studies of both mutant and wildtype enzymes for verification, which is usually beyond the research goals of most enzyme engineering laboratories. One of the most extensively studied enzymes that details the effect of mutations and 'double mutations' on the rates of individual kinetic steps and conformational equilibria as related to catalysis is the enzyme dihydrofolate reductase (DHFR). The extensive data and references to the individual studies on this enzyme are well summarized in a review by Miller and Benkovic [11].

The steady-state kinetic value that one sees most often in the literature on engineered or mutant proteins is that of $k_{\text {cat }} / K_{\mathrm{m}}$ as a measure of the catalytic efficiency of the enzyme and a measure of its catalytic usefulness relative to wild-type, other mutant forms of the enzyme, or of other biological sources of the same catalyst. The term $k_{\text {cat }} / K_{\mathrm{m}}$ has the units of a second-order rate constant (e.g. $M^{-1} \mathrm{~s}^{-1}$ ) (see Equation 1.2). Northrop has written a valuable discussion of the term $k_{\mathrm{cat}} / K_{\mathrm{m}}$ and its use in our understanding of relative catalytic efficiencies [12, 13]. In accord with his arguments, this term represents a 'capture rate' of the enzyme for its substrate, as this value represents all of the kinetic steps up to and including the first irreversible step in catalysis. In the simplest case of a reversible enzymatic reaction with one substrate, the kinetic parameters can be visualized as shown in Equation 1.3. The kinetic terms comprising $k_{\text {cat }}$ are given in Equation 1.4, which reduces to 
Equation 1.5 when the chemical step is fully rate-limiting in turnover, and reduces to Equation 1.6 when product release is rate-limiting.

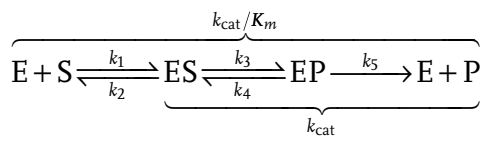

$$
\begin{aligned}
& k_{\text {cat }}=\frac{k_{3} k_{5}}{k_{3}+k_{5}} \\
& k_{\text {cat }}=k_{3} \\
& k_{\text {cat }}=k_{5}
\end{aligned}
$$

The expression for $k_{\text {cat }} / K_{\mathrm{m}}$ is given in Equation 1.7.

$$
\frac{k_{\mathrm{cat}}}{K_{\mathrm{m}}}=\frac{k_{1} k_{3} k_{5}}{k_{2}\left(k_{4}+k_{5}\right)+k_{3} k_{5}}
$$

In the situation where the chemical step is irreversible in catalysis, the expression describing this situation is shown in Equation 1.8. The expression for $k_{\text {cat }}$ in this case is the same as in the previous case, and shown in Equations 1.4-1.6. The expression for $k_{\text {cat }} / K_{\mathrm{m}}$ does differ from the above case, as shown in Equation 1.9 .

$$
\begin{aligned}
& \overbrace{\mathrm{E}+\mathrm{S}}^{\stackrel{k_{1}}{\rightleftharpoons} \underbrace{\mathrm{ES} \stackrel{\mathrm{k}_{3}}{\longrightarrow} \mathrm{EP} \underset{k_{\text {cat }}}{k_{\text {cat }} / K_{\mathrm{m}}} \mathrm{E}+\mathrm{P}}_{k_{2}}} \\
& \frac{k_{\text {cat }}}{K_{\mathrm{m}}}=\frac{k_{1} k_{3}}{k_{2}+k_{3}}
\end{aligned}
$$

In contrast, $k_{\text {cat }}$ represents all of the reaction steps in the catalytic cycle with the exclusion of the substrate-binding steps (as the substrate concentration is saturating), and is limited by the step which is rate-limiting in catalysis (i.e. the total rate of catalytic turnover can be no faster than the slowest step in the reaction sequence). Most commonly, $k_{\text {cat }}$ values are determined by the rate of product release (Equation 1.6), but in some instances are determined by the rate of the chemical transformation step in the enzyme reaction (Equation 1.5). It should be noted that $k_{\text {cat }}$ values represent rates when all of the catalytic binding sites are saturated with substrate, and therefore relative substrate binding steps cannot be included in the their comparative values. 


\subsection{1}

\section{$\mathrm{pH}$-Dependence of Steady-State Kinetic Parameters}

Generally, a key aspect in studying enzyme catalytic properties is to determine the influence of $\mathrm{pH}$ on kinetic parameters. In comparing wild-type and mutant enzyme activities, it is important to verify whether or not the mutation has altered $\mathrm{pH}-$ activity profiles. For correct comparison of mutant and wild-type catalytic activities, the data should be acquired over a $\mathrm{pH}$ range where kinetic parameters investigated are $\mathrm{pH}$-independent in order to avoid artifactual complications in the interpretation of the kinetic data. The variation of $k_{\text {cat }}$ with $\mathrm{pH}$ generally reflects alterations in the ionization state of essential amino acid residues in the active site that participate in catalysis. The variation of $k_{\text {cat }} / K_{\mathrm{m}}$ with $\mathrm{pH}$ may reflect either substrate binding $\left(\mathrm{p} K_{\mathrm{a}}\right.$ values due to either substrate or amino acid group at the binding site) or amino acid residues essential in the chemical step in catalysis. The point here is that engineering an enzyme may result in expected or unexpected alterations in the $\mathrm{p} K_{\mathrm{a}}$ of the amino acid side chains involved in substrate binding or in the chemical step in catalysis. Therefore, $\mathrm{pH}$-dependent catalytic characterization is an essential component of the characterization of engineered enzymes. The additional information obtained also would include $\mathrm{p} K_{\mathrm{a}}$ values for the participating amino acid residues, which are easily obtained from visual inspection and fitting of $\mathrm{pH}$-profile data with the appropriate equations.

\section{4 .2}

\section{Analysis of Two-Substrate Enzymes}

A general survey of known enzymes shows that a large majority of them catalyze reactions utilizing two substrates. The principal pathways discussed in most introductory biochemistry textbooks are the ternary complex mechanism (where both substrates or both products are bound to the enzyme catalytic site) or the binary or Ping-Pong mechanism (where only one substrate or product is bound at the catalytic site at any time during the catalytic reaction) (see Figure 1.4).

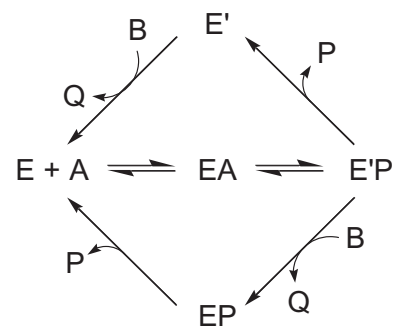

Figure 1.4 Two-substrate, two-product enzyme reaction pathway scheme depicting a binary (Ping-Pong) complex mechanism in the top loop and a ternary complex mechanism in the bottom loop. A and $B$ are the two substrates for the catalyzed reaction. $\mathrm{P}$ and $\mathrm{Q}$ denote the two products formed. 
It follows, therefore, that enzyme engineering might alter kinetic steps such that a two-substrate enzyme could follow a pathway different from that of the wild-type enzyme. The simplest case would be an alteration in the rate constant for release of the first product, resulting in reaction of the second substrate with the enzyme prior to release of the first product (or vice versa). It is therefore incumbent on the investigator not to assume retention of kinetic pathway on enzyme alteration but rather to verify whether any changes in fact did occur. This is readily shown by the variation of rates with different concentrations of both substrates to determine whether binary or ternary complex formation occurs through visual inspection (and fitting with the appropriate equations) of shapes of Lineweaver-Burk plots at different concentrations of the second substrate. The finding of intersecting Lineweaver-Burk plots demonstrates ternary complex behavior. If the plots are parallel, such behavior is suggestive of binary complex (or Ping-Pong) behavior, but does not unequivocally prove it as there are numerous examples in the literature of enzymes exhibiting parallel Lineweaver-Burk plots that, on further investigation, are shown to function by a ternary complex mechanism.

\section{5}

\section{Concluding Remarks}

The object of this chapter is to assist the protein engineer in the task of determining the functional properties of newly engineered enzymes. This task is not a trivial one, and in many instances represents far more effort and time than that spent on the construction of mutants and their expression. If the investigator adheres to the guidelines for the assay, accumulation of steady-state kinetic properties and their interpretations, as discussed in this chapter, then he or she should feel confident in further concepts and interpretations relevant to the field of enzyme engineering in which they are involved. The reader is also referred to excellent chapters dealing with more specialized aspects of the kinetic properties of enzyme systems in three volumes of Methods in Enzymology [14-16].

\section{References}

1 Cornish-Bowden, A. (2004) Fundamentals of Enzyme Kinetics, 3rd edn, Portland Press, London.

2 Cook, P.F. and Cleland, W.W. (2007) Enzyme Kinetics and Mechanism, Garland Science Publishing, New York.

3 Rudolph, F.B., Baughes, B.W. and Beissner, R.S. (1979) Methods in Enzymology, Part A, 63, Academic Press, New York, pp. 22-42.
4 Zhou, M., Diwu, Z., Panchuk-Voloshina N. and Hougland, R.P. (1997) Analytical Biochemistry, 253, 162-8.

5 Zhou, M. and Panchuk-Voloshina, N. (1997) Analytical Biochemistry, 253, 169-74.

6 Holt, A. and Palcic, M. (2006) Nature Protocols, 1, 2498-505.

7 Seiler, N., Jung, M.J. and Koch-Weser, J. (1978) Enzyme-Activated Irreversible Inhibitors, Elsevier Pub. Co., Amsterdam. 
8 Fowler, C.J., Wiberg, A., Oreland, L. and Wimblad, B. (1980) Neurochemical Research, 5, 697-708.

9 O’Farrell, N., Kreiner, M., Moore, B.D. and Parker, M.C. (2006) Biotechnology and Bioengineering, 95, 767-71.

10 Hubálek, F., Pohl, J. and Edmondson, D.E. (2003) The Journal of Biological Chemistry, 278, 28612-8.

11 Miller, G.P. and Benkovic, S.J. (1998) Chemistry and Biology, 5, R105-13.

12 Northrop, D.B. (1998) Journal of Chemical Education, 75, 1153-7.
13 Northrop, D.B. (1999) Enzyme Mechanisms, Vol. 27 (eds P.A. Frey and D.B. Northrup), IOS Press, Amsterdam, pp. 250-63.

14 Purich, D. (ed.) (1979) Methods in Enzymology, Part A, 63, Academic Press, New York.

15 Purich, D. (ed.) (1980) Methods in Enzymology, Part B, 64, Academic Press, New York.

16 Purich, D. (ed.) (1982) Methods in Enzymology, Part C, 87, Academic Press, New York. 
